American Journal of Infectious Diseases 4 (1): 76-84, 2008

ISSN 1553-6203

(C) 2008 Science Publications

\title{
Epitope Mapping of Dengue-Virus-Enhancing Monoclonal-Antibody Using Phage Display Peptide Library
}

\author{
${ }^{1}$ Chung-I Rai, ${ }^{2}$ Huan-Yao Lei, ${ }^{2}$ Yee-Shin Lin, ${ }^{2}$ Hsiao-Sheng Liu, ${ }^{2}$ Shun-Hua Chen, \\ ${ }^{3}$ Lien-Cheng Chen and ${ }^{1}$ Trai-Ming Yeh \\ ${ }^{1}$ Medical Laboratory Sciences and Biotechnology \\ ${ }^{2}$ Department of Microbiology and Immunology \\ ${ }^{3}$ Institute of Basic Medical Sciences, College of Medicine, \\ National Cheng Kung University, Tainan, Taiwan
}

\begin{abstract}
The Antibody-Dependent Enhancement (ADE) hypothesis has been proposed to explain why more severe manifestations of Dengue Hemorrhagic Fever and Dengue Shock Syndrome (DHF/DSS) occur predominantly during secondary infections of Dengue Virus (DV) with different serotypes. However, the epitopes recognized by these enhancing antibodies are unclear. Recently, antipre-M monoclonal antibody (mAb 70-21), which recognized all DV serotypes without neutralizing activity, were generated and demonstrated as an enhancing antibody for DV infection. In the present study, the epitope recognized by mAb 70-21 was identified using a phage-displayed random-peptide library. After three rounds of biopanning, ELISA showed that immunopositive phage clones specifically bound to mAb 70-21 but not to serum or purified IgG from naive mice. DNA sequencing of these phage clones showed a consensus sequence, QNNLGPR. Like mAb70-21, these phageinduced antisera also enhanced the DV infection of cells. In addition, indirect fluorescent assays showed phage-induced antisera bound to human rhabdomyosarcoma or Vero cells. Western blotting and immunoprecipitation analysis showed that phage-induced antisera recognized hsp 60 in BHK cell lysate. Moreover, the sera levels of antibodies against the synthetic peptide QNNLGPR correlated with the disease severity of dengue patients. Taken together, these results suggest that antibodies which recognized epitopes shared by pre-M of DV and hsp 60 of host cells may enhance DV infection and be involved in the development of DHF or DSS.
\end{abstract}

Key words: Dengue, Dengue Hemorrhagic Fever (DHF), Antibody-Dependent Enhancement (ADE)

\section{INTRODUCTION}

Dengue Virus (DV) is a single-stranded positivesense RNA virus in the flaviviridae family ${ }^{[1]}$. The mature virion consists of three structural (core, premembrane and envelope) and seven non-structural (NS1, NS2a, NS2b, NS3, NS4a, NS4b and NS5) proteins. DV is subgrouped into four antigenically related serotypes: $1,2,3$ and 4 . DV infection is the most important arboviral disease in humans, which can cause mild Dengue Fever (DF) as well as severe Dengue Hemorrhagic Fever/Dengue Shock Syndrome $(\mathrm{DHF} / \mathrm{DSS})^{[2,3]}$. An estimated 50 million dengue infections and 500,000 DHF cases occur annually, particularly in Southeast Asia, the western Pacific and the Americas ${ }^{[4]}$.

The majority of severe DV infections occur upon secondary infection with different serotypes of DV, suggesting an immune mediated mechanism in DHF/DSS. Various hypotheses for the pathogenesis of DHF/DSS have been proposed. The AntibodyDependent Enhancement (ADE) hypothesis emphasizes the importance of the immune response in the development of DHF/DSS ${ }^{[5-8]}$.

According to the ADE hypothesis, more severe manifestations of DHF/DSS occur predominantly, although not exclusively, in children with a second dengue virus infection and that a non-neutralizing antidengue antibody bound to a dengue virion increases the virus entrance into target cells, such as monocytes, macrophages, and B cells via the Fc receptor.

There are, however, several questions that remain to be solved before we fully understand the role of antibody in the development of DHF/DSS such as the significance of antibody specificity and the dependence of $\mathrm{Fc}$ receptor. Phage-display random-peptide libraries

Corresponding Author: Dr. Trai-Ming Yeh, Department of Medical Laboratory Sciences and Biotechnology,

College of Medicine National Cheng Kung University, Tainan 701, Taiwan 
have been used widely and successfully to rapidly identify and amplify peptide ligands for antibodies based on the affinity of their binding specificities ${ }^{[9]}$.

The B-cell epitope of DV type 1, which corresponded to amino acid residues 111-116 of the nonstructural protein 1 (NS1) of DEN-1, has been identified using a serotype-specific monoclonal antibody (mAb) of DV type 1 from a random peptide library displayed on phage ${ }^{[10]}$. DV neutralizing epitope has also been mapped used murine mAb 4E11, which can neutralize DV of all serotypes by binding to the 296-400 segment of the major E glycoprotein ${ }^{[11]}$. All these results indicate phage display peptide library can be a very powerful tool to identify ligands which $\mathrm{mAb}$ can specifically recognize. Here we used a phagedisplay random-peptide library in this study to identify the epitopes recognized by a monoclonal antibody (mAb 70-21) that enhances DV infection in vitro ${ }^{[12]}$.

\section{MATERIALS AND METHODS}

Producing hybridoma and purifying anti-pre-M mAb: The hybridoma cell line 70-21, which generates mAb 70-21 against all DV serotypes, was kindly provided by Dr. H.Y. Lei ${ }^{[12]}$. Hybridoma cells were seeded $\left(2 \times 10^{5}\right.$ cells $\left.\mathrm{mL}^{-1}\right)$ in Dulbecco's modified Eagle's minimum essential medium (DMEM; Invitrogen, Carlsbad, CA) supplemented with $10 \%$ fetal bovine serum (FBS; Invitrogen) and $1 \%$ penicillinstreptomycin-glutamine (Invitrogen) and then incubated at $37^{\circ} \mathrm{C}$.

After they had been incubated for 2 days, the culture supernatant was harvested and centrifuged at$1,000 \mathrm{rpm}$ for $10 \mathrm{~min}$ at $4^{\circ} \mathrm{C}$. The clarified supernatant was filtered $(0.45 \mu \mathrm{m})$ to remove cellular debris and IgG was purified using a protein $G$ affinity column (Amersham Pharmacia Biotech, Piscataway, NJ) and concentrated using Amicon ultra-15 centrifugal filter devices (Millipore, Bedford, MA). The final concentration of antibody was determined using a BCA assay (Bio-Rad Laboratories, Hercules, CA). The purity of the antibodies was analyzed using electrophoresis on a 10\% SDS-polyacrylamide gel (Sigma-Aldrich, St. Louis, MO). The protein in the gel was then detected using Coomassie Blue staining.

Affinity selection of phages using biopanning against mAb 70-21 with a phage-displayed random-peptide library: We used a phage-display random-peptide library kit (PhD 7-mer; New England Biolabs, Ipswich, MA) to identify epitopes recognized by mAb 70-21.
Phages bound to mAb 70-21 were isolated from the phage-display library using Protein $G$ sepharose beads (Amersham). The details of the affinity selection of phages using biopanning were as follows. Protein G sepharose beads (50\% aqueous suspension, $50 \mu \mathrm{L})$ were washed 3 times by spinning them for $30 \mathrm{sec}$ with $1 \mathrm{~mL}$ of TBS/0.1\% Tween 20 (TBST) and then suspended with $1 \mathrm{~mL}$ of BSA blocking buffer for $1 \mathrm{~h}$ at $4^{\circ} \mathrm{C}$ before they were used. Diluted phages from $10 \mu \mathrm{L}$ of the original library $\left(2 \times 10^{11}\right)$ were incubated with 10 $\mathrm{nM}$ of $\mathrm{mAb} 70-21$ in TBST for $20 \mathrm{~min}$ at room temperature. To increase the selection of peptides binding to mAb 70-21, we ran one round of negative selection with IgG from naive mice sera first.

Unbound phage particles were removed using centrifugation and washing, followed by three rounds of positive selection with mAb 70-21. Bound phages were eluted with glycine buffer ( $\mathrm{pH} 2.2$ ), neutralized with 1 $\mathrm{M}$ of Tris- $\mathrm{HCl}(\mathrm{pH} 9.1)$ and amplified for subsequent rounds of selection. Titration of phages in each step was done as follows: 10-fold serial dilutions of phages were made into Luria broth (LB). Each dilution $(10 \mu \mathrm{L})$ was incubated with $200 \mu \mathrm{L}$ of the midlog phase culture of Escherichia coli $\mathrm{ER} 2738\left(\mathrm{OD}_{600} \sim 0.5\right)$ for $5 \mathrm{~min}$ at room temperature and then plated with $3 \mathrm{~mL}$ top agarose onto LB/IPTG/Xgal agar plates. After incubating overnight at $37^{\circ} \mathrm{C}$, phage plaques were counted and the titer was calculated [phage titer $\left(\mathrm{pfu} 10 \mu \mathrm{L}^{-1}\right)=$ dilution factor $\times$ plaque number] and given in pfu $\mathrm{mL}^{-1}$.

Using ELISA to detect positive phage clones bound to mAb 70-21: We coated 96-well immunosorbent plates with $100 \mu \mathrm{L}$ of mAb 70-21 (100 $\left.\mu \mathrm{g} \mathrm{mL}^{-1}\right)$, irrelevant BSA, or serum or IgG from naive mice at $4^{\circ} \mathrm{C}$ overnight and then blocked them with $300 \mu \mathrm{L}$ of BSA blocking buffer for $2 \mathrm{~h}$ at room temperature. Approximately $10^{12}$ phage particles of the selected clones were added to each well and incubated for $1 \mathrm{~h}$ with shaking at room temperature. The plates were washed 6 times with TBST, incubated with $100 \mu \mathrm{L}$ of 1:5000 diluted horseradish peroxidase (HRP)conjugated anti-M13 antibody (Amersham) for $1 \mathrm{~h}$ and then washed 6 times with TBST. Bound HRPconjugated antibody were detected by adding $100 \mu \mathrm{L}$ of substrate tetramethylbenzidine (TMB) and terminated by adding $50 \mu \mathrm{L}$ of $2.0 \mathrm{M} \mathrm{H}_{2} \mathrm{SO}_{4}$. The absorbance was measured at $450 \mathrm{~nm}$ using an ELISA reader (Vmax microplate reader; Molecular Devices, Sunnyvale, CA).

Positive phage clones competitively inhibited mAb 70-21 from binding to DV-coated plates: For the competitive inhibition assay, diluted mAb 70-21 was 
pre-incubated with different concentrations of phages $\left(10^{3}-10^{6} \mathrm{pfu} \mathrm{mL}^{-1}\right)$ for $1 \mathrm{~h}$ at $37^{\circ} \mathrm{C}$ and then incubated with DV-coated plates $\left(10^{7}\right.$ antigen well $\left.{ }^{-1}\right)$ for $2 \mathrm{~h}$. After incubation, bound antibodies on the plates were detected by adding $100 \mu \mathrm{L}$ of 1:3,000-diluted HRP conjugated goat anti-mouse IgG (Sigma) for $1 \mathrm{~h}$. Color was developed by adding TMB and measured as described above.

Phage DNA sequencing and computer analysis: DNA sequences of purified phage clones were determined according to the dideoxynucleotide chain termination method using an automated DNA sequencer (ABI PRISM 377; Perkin-Elmer, Foster City, CA) with a BigDye Terminator cycle sequencing kit (version 3.1; Applied Biosystems). The primer used for phage DNA sequencing was 5'-CCCTCATAGTTAGCGTAACG-3'.

This primer is located in the antisense strand of gene III of the M13 phage and has 96 nucleotides separated from the inserted DNA. Inserted oligonucleotide sequences of phage DNA were selected and translated to peptide sequences. Peptide sequences were aligned using SeqWeb GCG programs (http://bioinfo.nhri.org.tw/gcg/index.htm) to find the conserved sequence with DV and other proteins.

Immunizing BALB/c mice with phage: $\mathrm{BALB} / \mathrm{c}$ mice (6 weeks old, female) from Laboratory Animal Center, National Cheng Kung University Medical College, Tainan, Taiwan, were immunized with phage. Before the immunization, the phages were UV-inactivated at $50 \mathrm{~mJ} \mathrm{~cm}{ }^{-2}$ for $1 \mathrm{~min}$. Phage particles were resuspended in PBS at a concentration of $10^{12} \mathrm{pfu}^{-1}$. Each phage clone $(200 \mu \mathrm{L})$ was intraperitoneally injected into four mice on days 0,7 and 14 . The mice were bled on day 21 .

Evaluating phage-induced antisera bound to DV: To analyze the binding activity of phage-induced antisera to DV, ELISA plates were coated with $100 \mu \mathrm{L}\left(10^{7}\right)$ per well of DV antigens and blocked with $300 \mu \mathrm{L}$ of blocking buffer. After blocking, each well was incubated with phage-induced antiserum or serum from naive mice (negative control) in a series of 10 -fold dilutions for $2 \mathrm{~h}$ at room temperature. Bound antibodies were detected by adding $100 \mu \mathrm{L}$ of HRP conjugated goat anti-mouse $\operatorname{IgG}$ (1:3,000 dilution) (Sigma) for $1 \mathrm{~h}$. Color was developed by adding TMB and read as described above.
Peptide inhibition experiments: The synthetic peptide QNNLGPR, which represents the inserted sequence of the selected positive-phage clones, was synthesized to see whether it inhibits phage-induced antisera from binding to DV. Briefly, phage-induced antisera (1:10,000 dilution) were pre-incubated with 10-fold increases in QNNLGPR $\left(0.01-100 \mu \mathrm{g} \mathrm{mL}^{-1}\right)$ or the control peptide GWGNGCGLFGKGGVVTCAKF $(\mathrm{D} 4 \mathrm{E})^{[13]}$ for $1 \mathrm{~h}$ at room temperature before they were incubated in DV-coated plates $\left(10^{7}\right.$ antigen well $\left.{ }^{-1}\right)$. Bound antibodies were detected by adding $100 \mu \mathrm{L}$ of HRP conjugated goat anti-mouse IgG (1:3,000 dilution) (Sigma) for $1 \mathrm{~h}$. The color was developed and read as described above.

Indirect immunofluorescence assay: Human rhabdomyosarcoma (RD) and Vero cell lines were cultured as previously described ${ }^{[14]}$. Aliquots of $100 \mu \mathrm{L}$ (approximately $1 \times 10^{5}$ cells) of RD and Vero cells from each experimental group were spun onto a slide using a cytocentrifuge at $1,000 \mathrm{rpm}$ for $10 \mathrm{~min}$, fixed with $4 \%$ paraformaldehyde in PBS and permeabilized with $0.1 \%$ saponin in PBS. After they had been blocked with blocking buffer, each slide was incubated with a 100 -fold dilution of phage-induced antiserum in a humidified chamber. After they had been incubated for $1 \mathrm{~h}$, antibodies bound to cells were detected by adding FITC-labeled goat anti-mouse IgG (Sigma) (1:64 dilution) in dilution buffer containing $0.01 \%$ Evans blue for $1 \mathrm{~h}$ in the dark. After they had been washed, the slides were mounted and observed under a fluorescent microscope (Olympus, Tokyo, Japan).

Western blot analysis: BHK-21 cells $\left(2 \times 10^{6}\right)$ scraped from culture plates were washed twice with PBS. The cells were then centrifuged at $1,000 \mathrm{rpm}$ for $5 \mathrm{~min}$ at $4^{\circ} \mathrm{C}$. The cell pellets were resuspended in $400 \mu \mathrm{L}$ of RIPA buffer (1\% NP-40, $0.5 \%$ sodium deoxycholate, $0.1 \%$ SDS, $10 \mathrm{mg} \mathrm{mL}^{-1}$ of PMSF, $30 \mathrm{~mL} \mathrm{~mL}^{-1}$ of aprotinin and $10 \mathrm{~mL} \mathrm{~mL}^{-1}$ of sodium orthovanadate in PBS). After the cells had been incubated on ice for 20 min, the protein lysates were collected using centrifugation at $13,000 \mathrm{rpm}$ for $30 \mathrm{~min}$ at $4^{\circ} \mathrm{C}$. Protein lysates $(10 \mu \mathrm{g})$ were separated using $10 \%$ SDS-polyacrylamide gel electrophoresis at $100 \mathrm{~V}$ for $90 \mathrm{~min}$ and transferred to a methanol activated polyvinylidene difluoride (PVDF) membrane (Hybond$\mathrm{P}$; Amersham) using a Trans-Unit semi-dry transfer cell (Amersham) at $60 \mathrm{~mA}$ for $1.5 \mathrm{~h}$ in a transfer buffer. The membranes were washed in PBST buffer for $5 \mathrm{~min}$ and then blocked in 5\% milk/PBST overnight. 
The next day, the membrane was incubated with phage-induced antiserum (1:100 dilution) at room temperature for $2 \mathrm{~h}$. After washing three times with PBST, the membrane was incubated with HRP-conjugated goat anti-mouse immunoglobulin (1:3,000 dilution) (Sigma) at room temperature for $1 \mathrm{~h}$. The proteins on the membrane were developed using an ECL chemiluminescence kit (Amersham).

Immunoprecipitation detecting of cellular protein bound to phage-induced antisera: $\mathrm{BHK}-21$ cells were lysed using RIPA buffer supplemented with protease and phosphatase inhibitor cocktails as described above. Cell lysates were incubated with phage-induced antiserum overnight at $4^{\circ} \mathrm{C}$. The resulting immunocomplex was precipitated with protein $G$ agarose for $4 \mathrm{~h}$. Proteins were eluted from the immunocomplex by boiling it for $10 \mathrm{~min}$ in $2 \mathrm{X}$ SDS sample buffer. Eluted proteins were resolved using $10 \%$ SDS-polyacrylamide gel and transferred to a PVDF membrane as described above.

The membranes were washed in PBST buffer for 5 min and then blocked in 5\% milk/PBST overnight. The next day, they were incubated with polyclonal goat anti-human hsp60 antibody (1:5,000 dilution) (Santa Cruz Biotechnology, Santa Cruz, CA) for $2 \mathrm{~h}$ at room temperature. After the membrane had been washed three times with PBST, it was treated with HRPconjugated rabbit anti-goat immunoglobulin $(1: 14,000$ dilution) (Sigma) at room temperature for $1 \mathrm{~h}$. The proteins bound with antibodies were detected using an ECL chemiluminescence kit.

Evaluating selected positive phage clones bound to anti-hsp60 antibody: ELISA plates were coated with 1 $\mu \mathrm{g}$ per well of goat anti-hsp60 antibody (Santa Cruz Biotechnology) and blocked with $300 \mu \mathrm{L}$ BSA blocking buffer. After they had been blocked, each well was incubated with selected positive phage clones or noninserted phages (controls) in a series of 10-fold dilutions for $2 \mathrm{~h}$ and $100 \mu \mathrm{L}$ of HRP conjugated antiM13 antibody (1:5,000 dilution) (Amersham) for $1 \mathrm{~h}$. After each step, wells were washed with PBS containing $0.1 \%$ Tween 20. Bound phages were detected by HRP-conjugated anti-M13 antibody and color developed as described above.

Plaque assay for antibody-dependent enhancement: BHK cells were seeded on 6 -well $\left(2 \times 10^{5}\right.$ cells well $\left.{ }^{-1}\right)$ plates in DMEM with $10 \%$ FBS for $3 \mathrm{~h}$ at $35^{\circ} \mathrm{C}$. When the cells had grown to confluent status, assays were performed by pre-incubating mAb 70-21 (1 $\mu \mathrm{g})$ or phage-induced antiserum (1:1,000 dilution) for $30 \mathrm{~min}$ at $4^{\circ} \mathrm{C}$, respectively. After they had been washed twice with PBS, a serial dilution of DV supernatants in DMEM with $2 \%$ FBS were added and the cells were incubated for $2 \mathrm{~h}$ at $37^{\circ} \mathrm{C}$. Subsequently, $2 \times \mathrm{DMEM}$ containing $2 \%$ low-melting-point agarose was added and the plates were incubated for 5 days at $37^{\circ} \mathrm{C}$. The plaques were measured as previously described ${ }^{[15]}$. Virus concentrations were determined as PFU per mililiter. The fold-enhancement of PFU was calculated by comparing it to PFU without antibody treatment.

Dengue patients and serum samples: During an outbreak of type $2 \mathrm{DV}$ in southern Taiwan (AugustOctober 2002) ${ }^{[16]}$, sera were obtained from 32 dengue patients in the acute stage of the disease between 1-18 days after fever onset. Twelve cases were classified as DF and 20 as DHF according to World Health Organization criteria. All 32 patients had high fever, headache and muscle and joint pain, but only the DHF patients were hospitalized for treatment. During this study, additional serum samples from normal adults ( $n=12$ ) without antibodies against dengue virus were obtained during routine health examinations and used as controls. All sera were collected and stored at $-80^{\circ} \mathrm{C}$ until used.

Detecting antibodies against the synthetic peptide QNNLGPR in sera from patients infected with dengue virus: ELISA plates were coated with $100 \mu \mathrm{L}$ $\left(10 \mu \mathrm{g} \mathrm{mL}^{-1}\right)$ per well of QNNLGPR and blocked with $300 \mu \mathrm{L}$ of blocking buffer. After blocking, each well was incubated with sera from patients infected with dengue virus and from normal controls at a 1:100 dilution for $2 \mathrm{~h}$ and $100 \mu \mathrm{L}$ of HRP conjugated goat anti-human $\operatorname{IgG}(1: 5,000$ dilution) (Sigma) for $1 \mathrm{~h}$. Bound antibodies were detected by color development as described above.

Statistical analysis: Data are expressed as mean \pm SD. The significance of the difference between the patient and control groups was analyzed using Student's t-test. Statistical significance was set at $\mathrm{p}<0.05$.

\section{RESULTS}

Screening mAb 70-21 binding phages with the phage-displayed peptide library: To screen phages that bind to mAb 70-21 in biopanning experiments, we first performed a negative selection against $\mathrm{IgG}$ from naive mice and then three positive selections against $\mathrm{mAb} 70-21$ to isolate phage clones specifically bound 
Table 1: Affinity selection of phage-display random-peptide library with $\mathrm{mAb} 70-21^{\mathrm{a}}$

\begin{tabular}{llll}
\hline $\mathrm{mAb}(70-21)$ & Input & Output & Recovery $(\%)$ \\
\hline Round 1 & $6.4 \times 10^{9}$ & $2.2 \times 10^{3}$ & $3.4 \times 10^{-5}$ \\
Round 2 & $8.1 \times 10^{11}$ & $6.8 \times 10^{5}$ & $8.3 \times 10^{-5}$ \\
Round 3 & $2.5 \times 10^{11}$ & $1.2 \times 10^{6}$ & $4.8 \times 10^{-4}$ \\
\hline
\end{tabular}

${ }^{a}$ : Biopanning was done as described in Materials and Methods using $100 \mu \mathrm{g} \mathrm{mL} \mathrm{m}^{-1} \mathrm{mAb} 70-21$. The percentage of recovery was calculated using (output titer/input titer) $\times 100 \%$
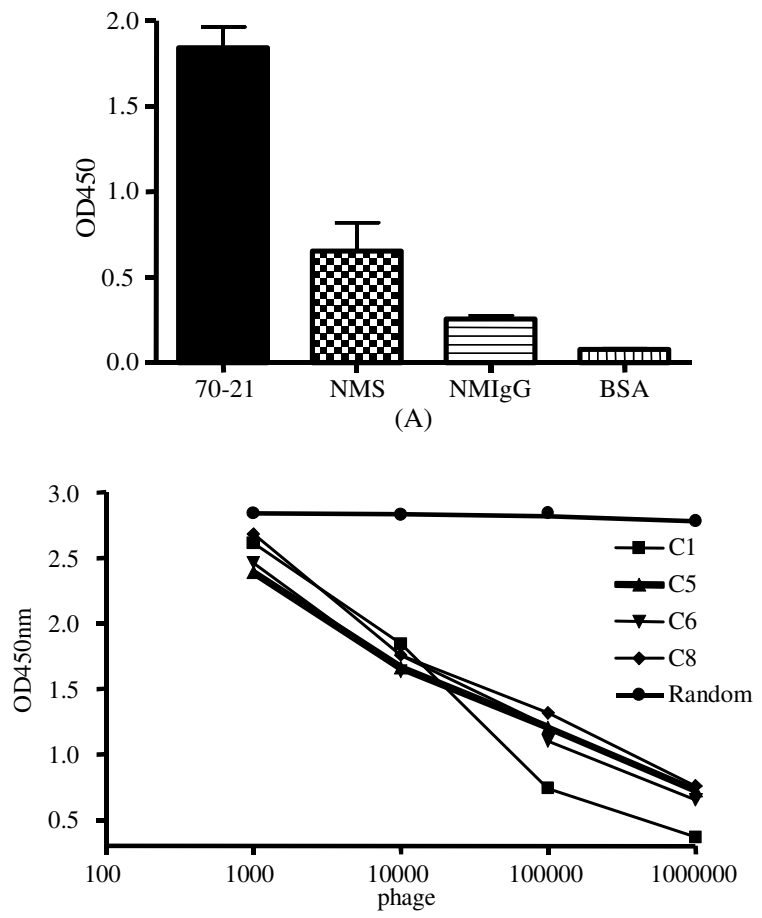

(B)

Fig. 1: Positive phage-clones bound specifically to monoclonal antibody 70-21 (mAb 70-21) and inhibited it from binding to Dengue Virus (DV). (A): Equal amounts $\left(100 \mu \mathrm{g} \mathrm{mL} L^{-1}\right)$ of $\mathrm{mAb}$ 70-21, naive mouse sera, naive mouse $\operatorname{IgG}$ and bovine serum albumin were immobilized in wells of ELISA plates. Selected positive phageclones $\mathrm{C} 1\left(10^{12}\right.$ plaque-forming units (pfu) were added to each well. Bound phages were detected using HRP-conjugated anti-M13 phage antibody and then substrate tetra-methyl benzidine (TMB) color development. Data expressed as mean \pm SD. (B): Different concentrations of positive phage-clones $(\mathrm{C} 1, \mathrm{C} 5, \mathrm{C} 6, \mathrm{C} 8)\left(10^{3}-10^{6} \mathrm{pfu}\right)$ were co-incubated with $\mathrm{mAb} 70-21$ for $30 \mathrm{~min}$ at $37^{\circ} \mathrm{C}$ and then transferred to the DV particlescoated plate. Bound mAb 70-21 were detected by adding HRP-conjugated goat anti-mouse IgG and substrate TMB to $\mathrm{mAb} 70-21$. After three rounds of biopanning, the ratio of output to input increased from $3.4 \times 10^{-5}$ to $4.8 \times 10^{-4}$, which suggested that specific enrichment had occurred (Table 1).

Ten phage clones (C1-C10) that showed significant binding to mAb 70-21 but not to naive mouse sera (NMS), naive mouse IgG (NMIgG), or bovine serum albumin (BSA) (Fig. 1A), were selected at random. To study their interaction with $\mathrm{mAb} 70-21$ further, we used competitive inhibition of $\mathrm{mAb} 70-21$ binding to DV-coated plates in the presence of phage clones. Increasing the concentration of positive phage clones (C1, C5, C6, C8) but not random phages caused a decrease in the amount of mAb 70-21 bound to dengue viral particles (Fig. 1B).

To understand the sequence of the peptide displayed by each positive phage clone, the phage single strand DNA was isolated and its nucleotide sequence was determined. We deduced an amino acid sequence (QNNLGPR) in the nucleotide sequences from all ten selected phages.

Study on the immunogenicity of selected positive phage clones: To further confirm that the selected phage clones indeed expressed peptide-mimicking dengue virus, two of the selected phage clones $(\mathrm{C} 5, \mathrm{C} 8)$ were injected into $\mathrm{BALB} / \mathrm{c}$ mice, in parallel with control phages (last panning residual pool; named random phages) and, using ELISA, the immune sera were tested for binding to their self-phage as well as to dengue-virus-coated plates. The immune sera elicited from the $\mathrm{C} 5$ phage (C5S)-, C8 phage (C8S)- and control phage (RS)-immunized mice reacted significantly to their corresponding phages compared to naïve mouse sera (data not shown).

In addition, we found more binding to dengue-virus-coated plates in C5- and C8-phageinduced antisera than in random phage-induced and naïve mouse sera (data not shown). To further confirm that QNNLGPR indeed mimicked dengue virus and was recognized by C5- and C8-phage-induced antisera, we used synthetic peptide QNNLGPR to competitively inhibit phage-induced antisera binding to dengue-viruscoated plates. We found that the synthetic peptide QNNLGPR but not the control peptide dosedependently inhibited the phage-induced antisera from binding with dengue virus particles (Fig. 2).

Analyzing putative cell-surface epitopes recognized by mAb 70-21 and phage-induced antisera: It has been found in previous study that mAb 70-21 recognizes self-antigen such as heat shock protein 60 on the cell surface and binds to BHK-21 and A549 cell ${ }^{[12]}$. 
Am. J. Infect. Dis., 4 (1): 76-84, 2008
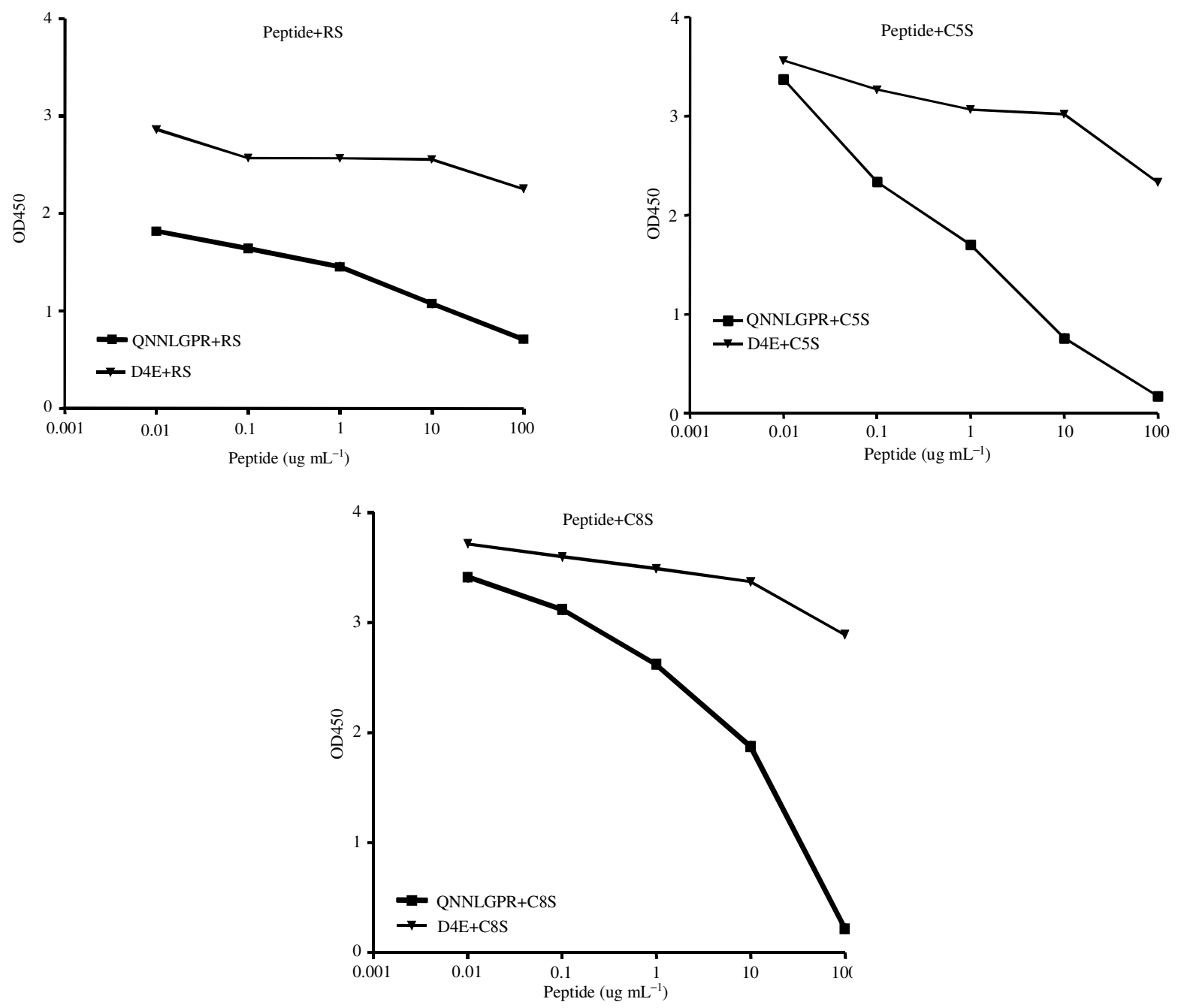

Fig. 2: The synthetic peptide QNNLGPR competitively inhibited positive-phage- but not random-phage-induced antisera from binding to dengue viral particles. Antisera against random phages (RS) and positive-phage clones C5 (C5S) or C8 (C8S) were incubated with different concentrations $\left(0.1-100 \mu \mathrm{g} \mathrm{mL}^{-1}\right)$ of the specific peptide QNNLGPR or unrelated D4E peptide for $1 \mathrm{~h}$ at room temperature before being incubated in denguevirus-coated plates $\left(10^{7}\right.$ antigen well $\left.{ }^{-1}\right)$. Bound antibody was detected by adding HRP-conjugated goat antimouse IgG and then substrate TMB

In the present study, we also found, by indirect fluorescent staining, that antisera against selected phage clones, but not random clones, cross-reacted against RD and Vero cells (Fig. 3). These results suggested that phage-induced antisera and mAb 70-21 may recognize a cellular protein expressed on different cells. To identify the cellular protein recognized by phageinduced antisera, we aligned QNNLGPR with human hsp60 by GCG program. We found a conserved motif (NxxGxR) shared by human hsp60 and QNNLGPR. To further confirm that phage-induced antisera recognized hsp60, the sera raised against phage clone 5 were used in Western blot and immunoprecipitation analyses. One apparent band with a molecular weight of about $60 \mathrm{kDa}$ of cell lysate was identified by Western blot (Fig. 4A) Moreover, immunoprecipitation assay demonstrated that phage-induced antisera immunoprecipitated a protein from BHK cell lysate which was also recognized by anti-hsp60-antibody (Fig. 4B). In addition, we found that the selected phage clones, but not random phage clones, could bind specifically to anti-hsp60-antibody-coated ELISA plates (Fig. 5). 
Am. J. Infect. Dis., 4 (1): 76-84, 2008

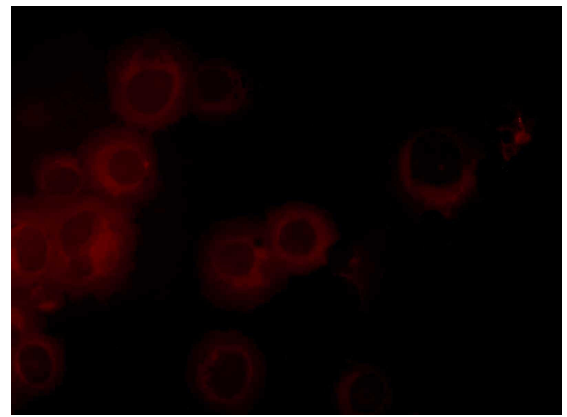

$\mathrm{RD}(\mathrm{RS})$

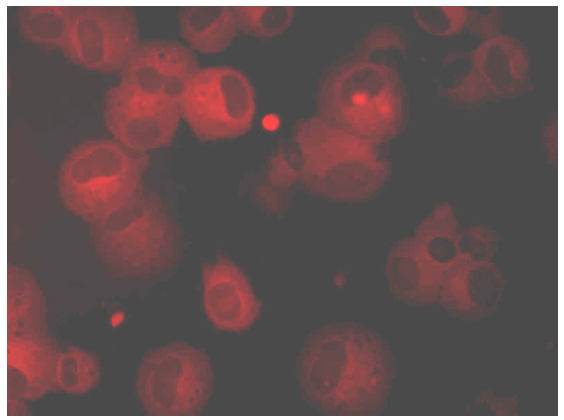

Vero (RS)

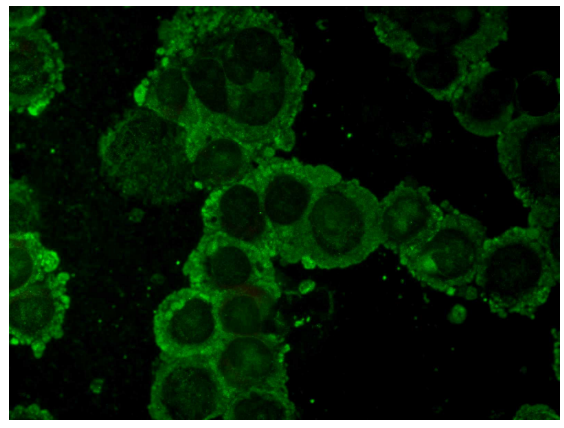

$\mathrm{RD}(\mathrm{C} 5 \mathrm{~S})$

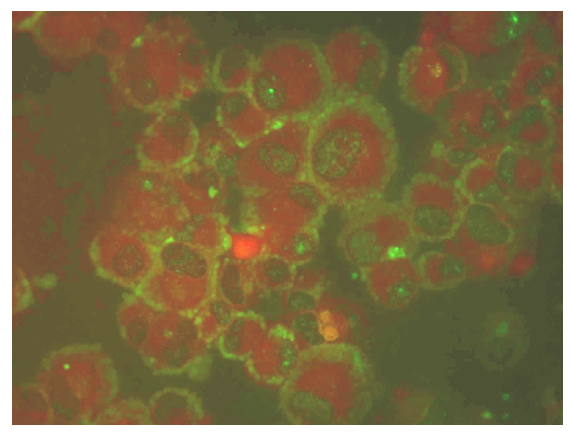

Vero (C5S)

Fig.3: Immunofluorescence staining analysis of of phage-induced antisera (C5S) bound to cells. Rhabdomyosarcoma (RD) and Vero cells were incubated with random phages-induced antisera (RS) or positive-phage clones C5-induced sera (C5S) as described in the Materials and Methods. The cells were observed under a fluorescent microscope. Antibodies bound to cells were detected by FITC-labeled goat antimouse IgG (Sigma) (Green). The cells were counterstained with Evans blue (red)

(A) Western blot (WB)

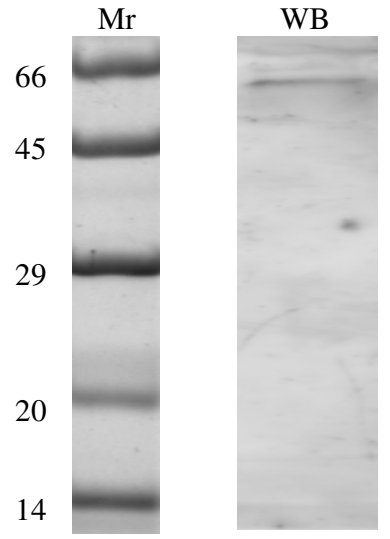

(B) Immunoprecipitation

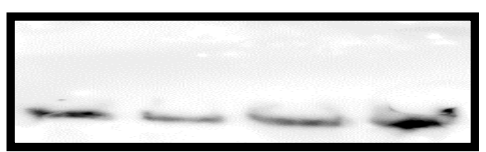

Fig. 4: Western blot and immunoprecipitation analyses of cell antigen recognized by phage-induced antisera. (A): BHK-21 cell lysates were separated using SDS-PAGE and transferred to PVDF membrane. After the cell lysates had been blocked, the membranes were incubated with phage-induced antiserum (C5S) as a primary antibody and then treated with HRP-conjugated goat anti-mouse IgG. Proteins recognized by antibodies were detected using enhanced chemiluminescence (ECL) substrate. (B): BHK-21 cell lysates were immunoprecipitated using phage-induced antisera (C5S) and subjected to 10\% SDS-PAGE and Western blot analysis. Anti-hsp 60 polyclonal antibodies were used to detect antigen in PVDF. Each lane represents one experiment 


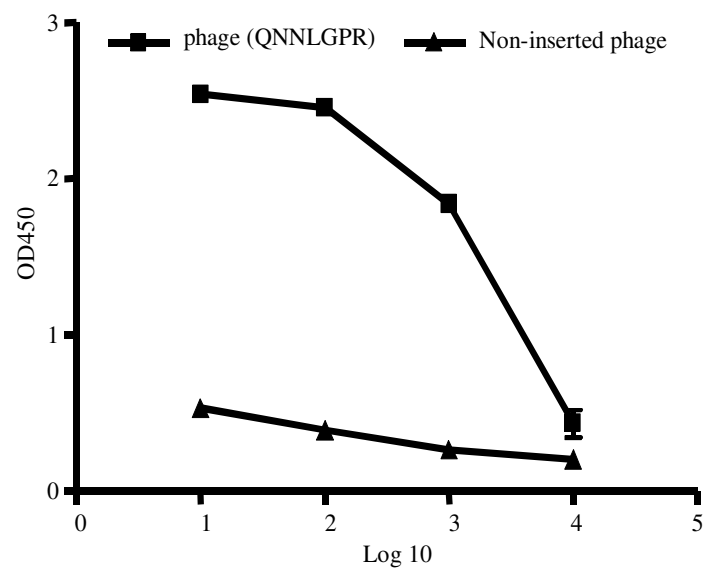

Fig. 5: Binding activity of selected phage C5 to hsp-60antibody-coated plates. Each well was coated with $1 \mu \mathrm{g}$ of hsp 60 antibody. Serially diluted C5 phages ( $\mathbf{m})$, which express QNNLGPR, or non-inserted phages $(\boldsymbol{\Delta})$ were added to hsp-60antibody-coated wells. Bound phages were detected using HRP conjugated anti-M13 phage antibody substrate TMB

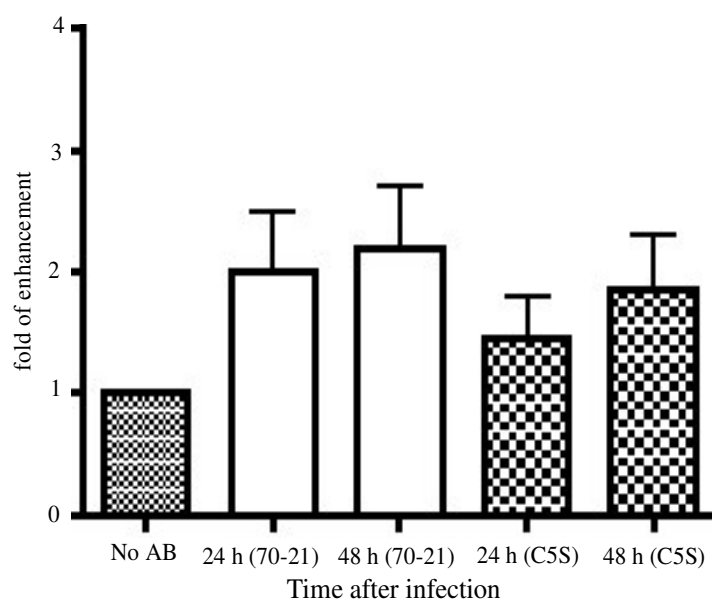

Fig. 6: Monoclonal antibody (mAb) 70-21 and C5 phage-induced antisera (C5S) enhanced Dengue Virus (DV) infection of BHK cells. BHK cells were grown to confluent status and preincubated with or without mAb 70-21 $(1 \mu \mathrm{g})$ or C5S (1:1,000 dilution) as indicated for $30 \mathrm{~min}$ at $4^{\circ} \mathrm{C}$. After washing, DV supernatants were added and incubated with the cells for $2 \mathrm{~h}$ at $37^{\circ} \mathrm{C}$. The virus titer was assayed $24 \mathrm{~h}$ or $48 \mathrm{~h}$ post-infection by plaque assay. Data expressed as mean $\pm \mathrm{SD}$

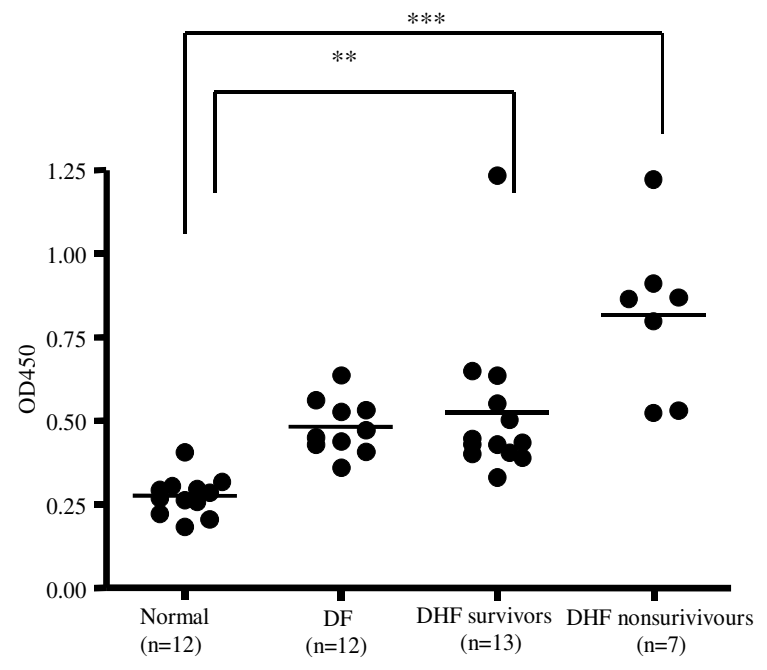

Fig. 7: ELISA analysis of antibody against synthetic peptide QNNLGPR in the sera of dengue patients. Sera from normal controls (Controls; $\mathrm{n}=12$ ), dengue fever (DF) patients $(\mathrm{n}=12)$ and dengue hemorrhagic fever patients (DHF Survivors; $\mathrm{n}=13$; DHF Nonsurvivors; $\mathrm{n}=7$ ) were assayed for their reactivity to synthetic peptide QNNLGPR (as described in the Materials and Methods). $* *: \mathrm{p}<0.01, * * *: \mathrm{p}<0.001$

Phage-induced antisera enhanced DV infection: To confirm that phage-induced antisera can enhance DV infection of cells as mAb 70-21 does, we used a plaque assay to examine whether phage-induced antisera and mAb 70-21 had the same enhancing effect on the DV infection of BHK-21 cells. BHK-21 cells were preincubated with $\mathrm{mAb} 70-21$ or phage-induced antisera before DV infection. We found that both phage-induced antisera and mAb 70-21 antibodies had comparable effects to enhance DV infection of BHK-21 cells (Fig. 6).

Antibodies against the synthetic peptide QNNLGPR in dengue patients' sera: To understand whether antibodies against the synthetic peptide QNNLGPR play a role in the pathogenesis of DV infection, we measured the sera levels of these antibodies in sera from dengue patients with different severity using an ELISA plate coated with synthetic QNNLGPR. We found a significant increase in the sera level of antibodies against this peptide in all dengue patients compared to sera from normal controls (Fig. 7). A positive correlation between the antibody titer and disease severity was also indicated. 


\section{DISCUSSION}

Selected phage clones which bound to dengue virus enhancing monoclonal antibody, $\mathrm{mAb} 70-21$ were isolated using phage-display random-peptide library. These phage clones could competitively inhibit $\mathrm{mAb}$ 70-21 binding to DV particles on ELISA plates. Furthermore, antisera against these phage clones mimicked mAb 70-21 binding to DV-coated plates and enhancing DV infection. These results suggested that the selected phage clones might have displayed a peptide which mimicked the epitope of dengue virus recognized by $\mathrm{mAb} 70-21$.

A conserved amino acid sequence QNNLGPR was found in the DNA sequence analysis of the nucleotides in the selected phage clones. In a previous study, we showed that $m A b$ 70-21 recognized a synthetic peptide corresponding to the M3 region (CPFLKQNEPEDIDCW) of the pre-M protein of $\mathrm{DV}^{[12]}$. Thus, based on these and our current findings, we speculate that the QNxxxP motif is important amino acid residues on DV to interact with mAb 70-21.

In addition to pre-M protein of DV, it has been shown previously that mAb 70-21 also cross-reacts to heat shock protein $60^{[12]}$, in the present study we found that NxxGxR sequence of QNNLGPR was also present in N-terminus amino acids 28-33 of human hsp60. This sequence homology between selected phage clones and hsp60 may explain why antisera against selected phage clones could cross-react with hsp60 as shown in immunoprecipitation assay. Furthermore, these hsp60 cross-reactive phage antibodies could bind to normal cells and enhance DV infection, probably through bridging together DV and host cells.

Last but not least, we found in this study there was a correlation between the sera levels of antibodies against the synthetic peptide QNNLGPR and the disease severity in dengue patients. Therefore, these results suggest antibodies against the synthetic peptide QNNLGPR can enhance DV infection and play an important role in the pathogenesis of DHF/DSS. Serological diagnosis using this synthetic peptide may provide diagnostic value for early identification of severe dengue patients, which might minimize DV morbidity and mortality.

\section{ACKNOWLEDGEMENT}

This study was supported by grant NHRI-CNCL9303P from the National Health Research Institute, Taiwan. We thank Bill Franke for editorial assistance.

\section{REFERENCES}

1. Henchal, E.A. and J.R. Putnak, 1990. The dengue viruses. Clin. Microbiol. Rev., 3: 376-396.

2. Halstead, S.B., 1989. Antibody, macrophages, dengue virus infection, shock and hemorrhage: a pathogenetic cascade. Rev. Infect. Dis., 11: S830 839 .
3. Halstead, S.B., 1982. Dengue: hematologic aspects. Semin. Hematol., 19: 116-131.

4. Kroeger, A., M. Nathan and J. Hombach, 2004. Dengue. Nat. Rev. Microbiol., 2: 360-361.

5. Halstead, S.B., 2003. Neutralization and antibodydependent enhancement of dengue viruses. Adv. Virus Res., 60: 421-467.

6. Halstead, S.B., 1970. Observations related to pathogensis of dengue hemorrhagic fever. VI. Hypotheses and discussion. Yale J. Biol. Med., 42: 350-362.

7. Halstead, S.B. and E.J. O'Rourke, 1977. Antibodyenhanced dengue virus infection in primate leukocytes. Nature, 265: 739-741.

8. Halstead, S.B., J.S. Porterfield and E.J. O'Rourke, 1980. Enhancement of dengue virus infection in monocytes by flavivirus antisera. Am. J. Trop. Med. Hyg., 29: 638-642.

9. Szardenings, M., 2003. Phage display of random peptide libraries: applications, limits and potential. J. Recept. Signal Transduct. Res., 23: 307-349.

10. Wu, H.C., Y.L. Huang, T.T. Chao, J.T. Jan, J.L. Huang, H.Y. Chiang, C.C. King and M.F. Shaio, 2001. Identification of B-cell epitope of dengue virus type 1 and its application in diagnosis of patients. J. Clin. Microbiol., 39: 977-982.

11. Thullier, P., C. Demangel, H. Bedouelle, F. Megret, A. Jouan, V. Deubel, J.C. Mazie and P. Lafaye, 2001. Mapping of a dengue virus neutralizing epitope critical for the infectivity of all serotypes: insight into the neutralization mechanism. J. Gen. Virol., 82: 1885-1892.

12. Huang, K.J., Y.C. Yang, Y.S. Lin, J.H. Huang, H.S. Liu, T.M. Yeh, S.H. Chen, C.C. Liu and H.Y. Lei, 2006. The dualspecific binding of dengue virus and target cells for the antibody-dependent enhancement of dengue virus infection. J. Immunol., 176: 2825-2832.

13. Huang, Y.H., B.I. Chang, H.Y. Lei, H.S. Liu, C.C. Liu, H.L. Wu and T.M. Yeh, 1997. Antibodies against dengue virus $\mathrm{E}$ protein peptide bind to human plasminogen and inhibit plasmin activity. Clin. Exp. Immunol., 110: 35-40.

14. Weng, T.Y., L.C. Chen, H.W. Shyu, S.H. Chen, J.R. Wang, C.K. Yu, H.Y. Lei and T.M. Yeh, 2005. Lactoferrin inhibits enterovirus 71 infection by binding to VP1 protein and host cells. Antiviral Res., 67: 31-37.

15. Huang, Y.H., H.Y. Lei, H.S. Liu, Y.S. Lin, C.C. Liu and T.M. Yeh, 2000. Dengue virus infects human endothelial cells and induces IL-6 and IL-8 production. Am. J. Trop. Med. Hyg., 63: 71-75.

16. Chen, L.C., H.Y. Lei, C.C. Liu, S.C. Shiesh, S.H. Chen, H.S. Liu, Y.S. Lin, S.T. Wang, H.W. Shyu and T.M. Yeh, 2006. Correlation of serum levels of macrophage migration inhibitory factor with disease severity and clinical outcome in dengue patients. Am. J. Trop. Med. Hyg., 74: 142-147. 